\title{
The Alpha-2A-Adrenoceptor Agonist, Guanfacine, Increases Regional Cerebral Blood Flow in Dorsolateral Prefrontal Cortex of Monkeys Performing a Spatial Working Memory Task
}

Robert A. Avery, M.A., Jenna S. Franowicz, M.Phil., Colin Studholme, Ph.D., Christopher H. van Dyck, M.D., and Amy F.T. Arnsten, Ph.D.

Research indicates that norepinephrine enhances the working memory functions of the prefrontal cortex (PFC) through actions at post-synaptic, alpha-2A adrenoceptors. The current study examined the effects of the alpha- $2 \mathrm{~A}$ adrenoreceptor agonist, guanfacine $(0.7 \mathrm{mg} / \mathrm{kg}, i . \mathrm{m}$.), compared to saline on SPECT measures of regional cerebral blood flow ( $r C B F)$ in monkeys performing a spatial working memory task. Animals were infused with the SPECT blood flow tracer, Tcm-99m ECD, through an indwelling intravenous catheter while performing the working memory task. Guanfacine treatment significantly improved cognitive performance of the working memory task, and significantly increased $r C B F$ values in the dorsolateral PFC, the brain region most tightly associated with performance of spatial working memory tasks. In contrast, guanfacine had no significant effect on $\mathrm{rCBF}$ in the superior temporal cortex, an auditory association area unrelated to task performance. These data are consistent with the hypothesis that alpha- $2 \mathrm{~A}$ adrenoceptor stimulation preferentially enhances functioning of the PFC. [Neuropsychopharmacology 23:240-249, 2000] (C) 2000 American College of Neuropsychopharmacology. Published by Elsevier Science Inc. All rights reserved.
KEY WORDS: Norepinephrine; Alpha-2 adrenergic receptors; Cognition; SPECT imaging; Working memory; Guanfacine

Evidence from a variety of disciplines indicates that norepinephrine (NE) has a critical beneficial influence on the working memory functions of the prefrontal cor-

From the Department of Diagnostic Radiology (RAA, CS) , Section of Neurobiology (JSF, AFTA), Department of Electrical Engineering (CS), and Department of Psychiatry (CHvD), Yale Medical School, New Haven, CT.

Address correspondence to: Dr. Amy F.T. Arnsten, Section of Neurobiology, Box 208001, Yale Medical School, 333 Cedar St., New Haven, CT 06520-8001.

Received August 3, 1999; revised March 2, 2000; accepted March 7, 2000 tex (PFC) through its actions at post-synaptic, alpha-2A adrenergic receptors (reviewed in Arnsten et al. 1996). In monkeys, the ability to perform spatial working memory tasks has been closely linked to the dorsolateral PFC (dlPFC) surrounding the principal sulcus, especially the caudal two thirds of the principal sulcal region (Goldman and Rosvold 1970; Goldman-Rakic 1987).

Alpha-2 adrenergic agonists such as clonidine, guanfacine, and medetomidine can improve spatial working memory performance in monkeys (Arnsten et al. 1988), rats (Carlson et al. 1992), and humans (Coull et al. 1995; Jakala et al. 1999), but have little effect or impair tasks dependent on posterior cortices or hippocampus (reviewed in Arnsten 1998). Dose response curves are con- 
sistent with beneficial actions at post-synaptic alpha-2 receptors, as the drugs become more potent and more efficacious in animals and patients with NE depletion (Arnsten and Goldman-Rakic 1985; Cai et al. 1993; Franowicz and Arnsten 1999; McEntee and Mair 1990). However, very high doses of guanfacine have recently been found to significantly improve spatial working memory performance in normal adult monkeys as well (Franowicz and Arnsten 1998). It is likely that the alpha-2A receptor subtype underlies guanfacine's beneficial effects on working memory function (Arnsten et al. 1988; Franowicz et al. 1998; Tanila et al. 1999), and guanfacine is currently the most selective alpha-2A agonist available (Uhlen and Wikberg 1991).

Several findings suggest that alpha-2 agonists improve, while alpha-2 antagonists impair, working memory function through direct actions in the PFC. For example, infusion of the alpha- 2 antagonist, yohimbine, into the monkey PFC produces a delay-related impairment in working memory, whereas alpha- 1 and beta adrenergic antagonists are without effect $(\mathrm{Li}$ and Mei 1994). Similarly, electrophysiological studies show that iontophoretic application of yohimbine onto PFC neurons suppresses delay-related activity, the cellular measure of working memory (Li et al. 1999; Sawaguchi 1998). Conversely, iontophoretic or systemic administration of clonidine increases delay-related firing, and this enhancement is reversed by iontophoretic application of yohimbine ( $\mathrm{Li}$ et al. 1999). Similarly, infusion of the alpha-2 agonist medetomidine, into aged rat PFC improves spatial working memory performance (Tanila et al. 1996).

Very few studies have taken advantage of imaging technologies to examine NE alpha-2 mechanisms in the cortex. Most human studies have utilized clonidine in which the potent side effects have generally limited research to low doses. Low doses of clonidine in healthy individuals likely engage predominantly presynaptic alpha-2 receptors and thus may produce widespread decreases in NE transmission. Low doses of clonidine $(0.1-10 \mathrm{ug} / \mathrm{kg}$ ) have little effect in normal young adult monkeys, but enhance working memory performance in monkeys with NE depletion (Cai et al. 1993; Franowicz and Arnsten 1999). Coull et al. (1997) using positron emission tomography (PET) found that low doses of clonidine $(1.3 \mu \mathrm{g} / \mathrm{kg})$ did not enhance blood flow in the PFC and indeed reduced regional cerebral blood flow $(\mathrm{rCBF})$ in thalamus during conditions of rest. Moffoot et al. (1994) examined the effects of this same dose of clonidine in Korsakoff's patients with presumed NE depletion. Previous research has shown that Korsakoff's patients have NE loss which correlates with memory impairment, and that clonidine can improve memory performance in these patients (Mair and McEntee 1986). Indeed, clonidine's efficacy in these patients correlated with indices of NE loss, consistent with drug actions at post-synaptic alpha-2 receptors (McEntee and Mair 1990). Moffoot and colleagues (1994) used single photon emission computed tomography (SPECT) imaging to examine changes in $\mathrm{rCBF}$ associated with clonidine vs. placebo treatment in Korsakoff's patients. Patients performed a word fluency task during the infusion of the SPECT tracer. Clonidine improved performance of the word fluency task, and this improvement correlated with increased $\mathrm{rCBF}$ in the left $\mathrm{PFC}$, the brain region most associated with performance of this task (Moffoot et al. 1994). This study also found that clonidine increased $\mathrm{rCBF}$ in the anterior cingulate cortex, although this finding did not correlate with changes in cognitive performance. This study was the first to suggest that alpha-2 agonist treatment can increase $\mathrm{rCBF}$ in the PFC during performance of a PFC task.

The current study used a similar approach to address this issue in monkeys, where effective doses for alpha-2 agonist treatment are well-established (Franowicz and Arnsten 1998). Animals were treated with saline or guanfacine $(0.7 \mathrm{mg} / \mathrm{kg}$, i.m. two hours before completing a test of spatial working memory, the delayed response task. During delayed response testing, animals were infused with the SPECT blood flow tracer, Technetium-99m ethyl cysteinate dimer (ECD), through an indwelling intravenous catheter. SPECT imaging has several advantages in that monkeys can be used as their own controls to compare the effects of saline versus guanfacine. Furthermore, the SPECT rCBF tracers can be injected during a task without disturbing the subject; the stability of the SPECT tracer permits the animal to be imaged after task completion and while anesthetized.

We hypothesized that guanfacine would increase rCBF in the dIPFC- specifically, the caudal two-thirds of the principal sulcal cortex most associated with performance of spatial working memory tasks. We, thus, examined two slices through the caudal two-thirds of the principal sulcal cortex: the caudal third of the principal sulcal cortex, hereafter referred to as caudal dlPFC, and the more rostral third mid-way through the principal sulcal cortex, hereafter referred to as mid dlPFC. We, further, proposed that guanfacine would have little effect on $\mathrm{rCBF}$ in a cortical region not relevant to the task. As auditory information is irrelevant to the performance of a spatial working memory task, and as alpha-2 agonists have been shown to have little effect on performance of temporal lobe tasks (Arnsten 1998), the superior temporal gyrus (STG; auditory association cortex) was selected a priori for comparison to the dlPFC. The results of this study may have direct clinical relevance, as guanfacine is being considered for use in neuropsychiatric disorders with prevalent PFC dysfunction such as Attention Deficit Hyperactivity Disorder (Chappell et al. 1995; Horrigan and Barnhill 1995; Hunt et al. 1995). 


\section{METHODS}

\section{Subjects}

The animals used in this study were four young adult female rhesus monkeys (Macaca mulatta) ranging in age from 6 years (post-pubescent) to 12 years (middle aged). The monkeys were individually housed and maintained on a diet of Purina monkey chow supplemented with fruit. Care of the animals followed the guidelines in "Guide For the Care and Use of Laboratory Animals" and was approved by the Yale Animal Care and Use Committee. Animals were always tested at the same time of day immediately prior to feeding. Highly palatable food rewards (e.g., peanuts, raisins, or chocolate chips) were utilized during testing to minimize the need for dietary regulation.

\section{Cognitive Testing}

Testing occurred in a Wisconsin General Testing Apparatus (WGTA) situated in a sound-attenuating room. Background masking noise (60 dB, wideband) was also used to minimize auditory distractions. The monkeys had been trained on the 2-well delayed response task as described previously (Arnsten et al. 1988). During delayed response, the animal watches as the experimenter baits one of two foodwells with a food reward. The foodwells are then covered with identical cardboard plaques, and an opaque screen is lowered between the animal and the tester for a specified delay. At the end of the delay, the screen is raised and the animal is allowed to choose. Reward is quasi-randomly distributed between the left and right wells over the 30 trials that make up a daily test session. Five different delay lengths (referred to as delays A through E) were also quasi-randomly distributed over these 30 trials.

\section{Drug Administration}

Animals were administered saline or guanfacine $(0.7$ $\mathrm{mg} / \mathrm{kg}$ ) intramuscularly 2-2 1/2 hours prior to testing. Two of the four received guanfacine first, and the other two guanfacine later. Drug was dissolved in sterile saline immediately prior to injection. Guanfacine was generously provided by Wyeth-Ayerst.

\section{Chair Training}

In order to inject the SPECT blood flow tracer during cognitive testing, the four animals needed to be trained to test while restrained in a primate chair, and with a catheter placed in their leg. These animals had previously been trained to perform cognitive testing while in a transport cage which did not limit their movement. Training them to test in the chair with their legs restrained and a catheter in place in one leg caused signif- icant disruption in task performance. It took an average of $6.5 \pm 1.5$ months for the animals to resume testing in a stable manner and thus be ready for imaging. It is due to this significant time needed to train each animal that the total number of subjects in the experiment was limited to four animals.

\section{Injection Procedure}

On the day of imaging, the animal was injected with saline or guanfacine $(0.7 \mathrm{mg} / \mathrm{kg}) 2-21 / 2$ hours prior to testing. Fifteen minutes prior to testing, the animal was chaired and an intravenous catheter was inserted into the saphenous vein. When the tester indicated that the animals delayed response performance seemed stable, 6 $\mathrm{mCi}(222 \mathrm{MBq})$ of ECD were injected via catheter over about 2 minutes (slow infusion). The slow bolus was used, as in human studies, to minimize venous extension and potentially distracting biological perceptions and responses, and to extend the levels of ECD in the brain over a wider time period that would include several trials. Each animal was tested on the same set of 11 trials, immediately following tracer injection. ECD peaks in the brain after about 20 seconds of injection (Walovitch et al. 1989), although the uptake interval between injection and imaging is irrelevant because the regional rates of elimination appear to be uniform. ECD provides excellent contrast between gray and white matter (ibid). Each animal was injected once following saline treatment and once following guanfacine treatment. After testing the animal for at least another five minutes, the experimenter removed the animal from the testing chamber and injected the monkey with a mixture of ketamine and atropine. When the animal was anesthetized, it was removed from the chair and transported to the imaging facility, where it was intubated and maintained on halothane gas for the duration of the scan.

\section{SPECT Acquisition}

Subjects were imaged within 45 minutes of the ECD injection on a brain dedicated SPECT camera with resolution in all three axes of 7-8 $\mathrm{mm}$ full width at half maximum (FWHM). Three animals were imaged on a three-headed Picker Prism 3000XP camera (Cleveland Heights, OH) using high resolution fan beam collimators. One animal was imaged on a CERASPECT camera (CERASPECT, Digital Scintigraphics, Waltham, MA). Transaxial slices were reconstructed using a conventional clinical filtered backprojection. Prefiltering was employed using an apodization filter with a cut-off frequency referenced to the image power spectrum. Due to the small size of the brain, no attenuation correction was performed. 


\section{MRI Acquisition}

MRI acquisition took place on a separate day from SPECT imaging. The animals were injected with ketamine/atropine and transported to the imaging facility, where they were maintained on halothane anaesthesia. All subjects had magnetic resonance imaging (MRI) on a GE Signa scanner (General Electric Medical Systems, Milwaukee, WI) with a 1.5 Tesla field strength. T-1 weighted $1.5 \mathrm{~mm}$ 3-dimensional volumetric spoiled gradient-echo (TR/TE, $23 / 5 \mathrm{msec}$ ) images with a $18 \mathrm{~cm}$ field of view were acquired in the coronal orientation. T-1 weighted images provide excellent the contrast between gray and white matter. Due to the response of the imaging coil, images of subjects 3 and 4 contained appreciable intensity inhomogeneity across the imaged field. To aid in registration and visual inspection an intensity uniformity correction was applied to these images.

\section{SPECT and MRI Image Processing}

The saline and guanfacine SPECT scans were rigidly aligned with the subjects MRI by multi-resolution optimization (Studholme et al. 1997) of normalized mutual information (Studholme et al. 1999). This fully automated technique seeks a rigid transformation which maximises a measure of information content of the combined MRI-SPECT image pair. The spatial distribution of image values is such that the maximum of this information measure corresponds to the maximum overlap of values representing air, gray, and white tissues in the two modalities. The approach has been shown to provided sub-voxel multi-modality alignment accuracy in human studies relative to estimates from bone implanted markers (West et al. 1997).

Following estimation of rigid alignment parameters, all three scans could then be viewed in a common coordinate system allowing for an accurate comparison between homologous brain regions. MRI-SPECT alignment was verified by careful inspection of interactively selected iso-contours from SPECT overlayed onto MRI in three orthogonal planes (Fitzpatrick et al. 1998).

To account for differences in SPECT tracer dose, corresponding intensity values in the saline and guanfacine scans were then normalized. This was achieved by evaluating the ratio of mean counts within an ROI drawn bilaterally in the occipital cortex (primary visual cortex). The occipital cortex was selected due to its very low noradrenergic innervation (Lewis and Morrison 1989). A pixel by pixel subtraction was then performed on the normalized values, illustrating increases and decreases in rCBF relative to the monkey's saline. This difference image could then be viewed and subsequently superimposed onto the subjects MRI to verify anatomic location of any local changes. Differences in rCBF can then be quantified as a percent change relative to the sa- line scan. All computer processing was carried out on a personal computer driven by Pentium $\mathrm{III}^{\mathrm{TM}}$ processor.

\section{SPECT and MRI Image Analysis}

The images were rotated so the inferior portion of the occipital lobe was parallel to the most anterior portion of the prefrontal cortex. Two investigators identified the following brain regions on each subject's MRI: a) mid dIPFC; b) caudal dlPFC; and c) STG perpendicular to the intersection of the corpus callosum and fornix. Analysis was restricted to these three cortical regions given the small number of subjects reducing statistical power for multiple comparisons. Bilateral ROIs were drawn on each area and then quantified to yield the percent $\mathrm{rCBF}$ change (see Figure 1). Figure 2 visualizes the subjects caudal dlPFC as seen on the saline SPECT scan, guanfacine SPECT scan, MRI, and the results of the SPECT image subtraction superimposed on the subjects MRI.

\section{Statistical Analysis}

All statistical analyses used within subjects comparisons. Cognitive testing data were analyzed using a twotailed paired T-test, also known as $\mathrm{T}$ dependent test (Tdep), whereby each subject's performance on saline was compared to their performance on guanfacine. Cognitive data were analyzed for the entire test session and for the 11 trials associated with ECD infusion.

SPECT imaging data were analyzed using a two-way analysis of variance with repeated measures (2-ANOVA$\mathrm{R})$ with factors of cortical region (mid dlPFC, caudal dIPFC, STG) and drug (saline or guanfacine). Planned comparisons (test of effects) examined: 1) possible differences in $\mathrm{rCBF}$ between cortical regions following saline administration; and 2) the effects of guanfacine vs. saline for each cortical region.

Drug-induced changes in cognitive performance (entire session) and in rCBF (caudal dIPFC) were correlated using a Pearson's r-test. Statistical analyses were performed on a Power Macintosh G3 using Systat software.

\section{RESULTS}

\section{Spatial Working Memory Performance}

Administration of guanfacine $(0.7 \mathrm{mg} / \mathrm{kg})$ improved performance on the spatial delayed response task in all four monkeys (Figure 3). Guanfacine produced varying levels of improvement across the entire session, producing an average \pm S.E.M. improvement of $15.1 \pm$ $5.7 \%$ (range of improvement from 1.7 to $22.9 \%$ increase in performance). As illustrated in Figure 3, the average \pm S.E.M. performance on saline across the entire session was $65.9 \pm 5.6 \%$ correct; the average \pm S.E.M. per- 


\section{ROI Placements}

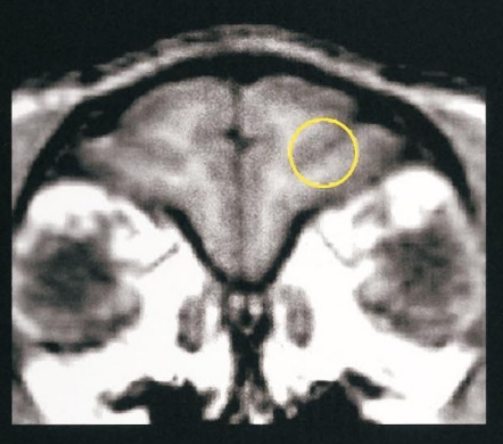

mid - PFC

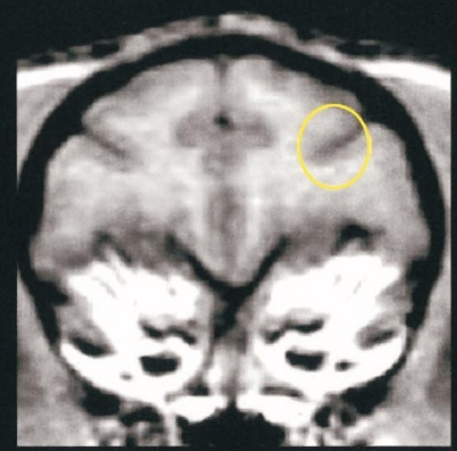

caudal - PFC

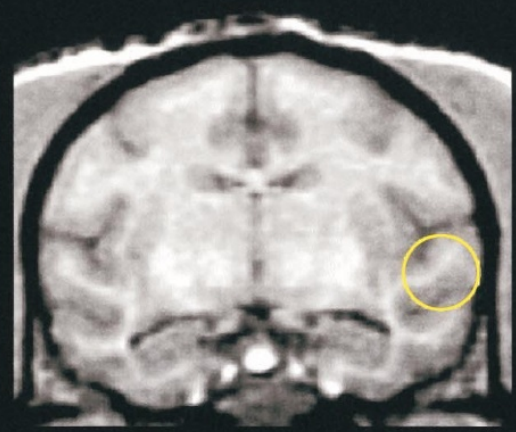

STG

Figure 1. The three regions of interest (ROIs) for the mid dlPFC, caudal dlPFC, and STG (auditory association cortex) are indicated by the yellow circles on coronal slices through the monkey brain. Although the ROIs are depicted unilaterally, the analyses were performed bilaterally. The right side of the monkey's brain is shown on the left side of the image.

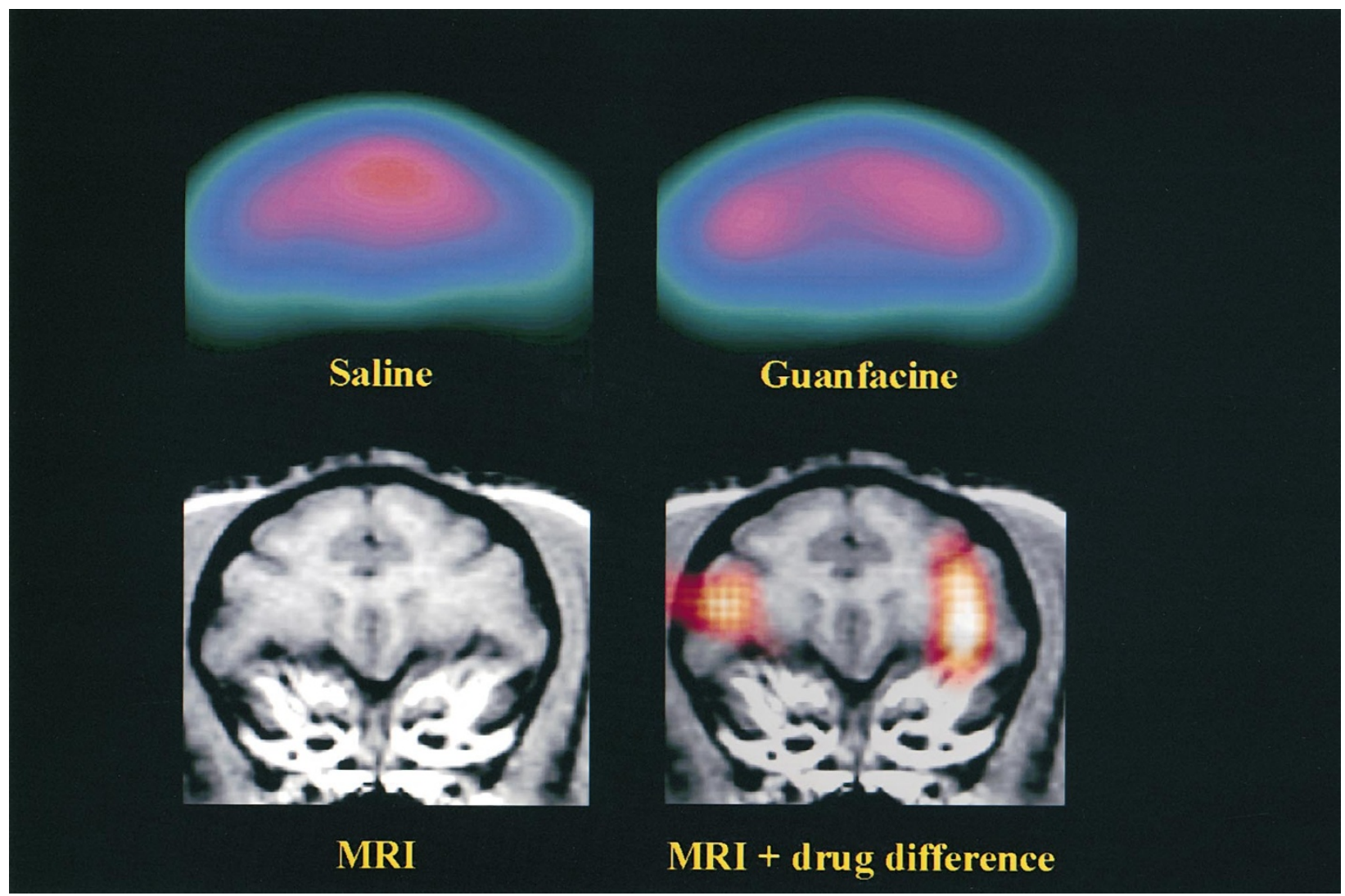

Figure 2. Composite figure illustrating the saline and guanfacine SPECT scans (upper left and right figures, respectively) and MRI scan (lower left figure) of the caudal PFC for monkey 1. The figure on the lower right depicts the difference between the saline and guanfacine SPECT scans overlaid on the MRI. The right side of the monkey's brain is shown on the left side of the image. Guanfacine significantly increased rCBF values in the dlPFC (see text). 
formance on guanfacine was $80.9 \% \pm 4.0 \%$ correct (saline vs. guanfacine: Tdep $=3.03, \mathrm{df}=3, p=.05$ ). Guanfacine consistently improved performance on the subset of 11 trials associated with ECD infusion, producing an average \pm S.E.M. improvement of $20.5 \pm$ $2.6 \%$ (range of 18.2 to $27.3 \%$ increase in performance, with three of the four monkeys exhibiting the $18.2 \%$ increase). As shown in Figure 3, the average \pm S.E.M. performance on saline for the subset of 11 trials was $61.4 \pm$ $2.6 \%$ correct, whereas the average \pm S.E.M. performance on guanfacine was $81.8 \pm 4.3 \%$ correct (saline vs. guanfacine: Tdep $=9.00, \mathrm{df}=3, p<.003$ ).

\section{SPECT Imaging}

SPECT data were analyzed using a two way analysis of variance with repeated measures with factors of cortical region (mid dlPFC, caudal dlPFC, STG) and drug (saline or guanfacine). Results showed a significant main effect of cortical region $(\mathrm{F}(2,6)=9.603, p=.013)$, a trend for main effect of drug $(\mathrm{F}(1,3)=9.645, p=.053)$, and a significant region by drug interaction $(\mathrm{F}(2,6)=$ $6.633, p=.03)$.

\section{Differences between Cortical Regions}

Although there was an overall significant main effect of cortical region, planned comparisons between saline scans for the three cortical regions failed to achieve sig-

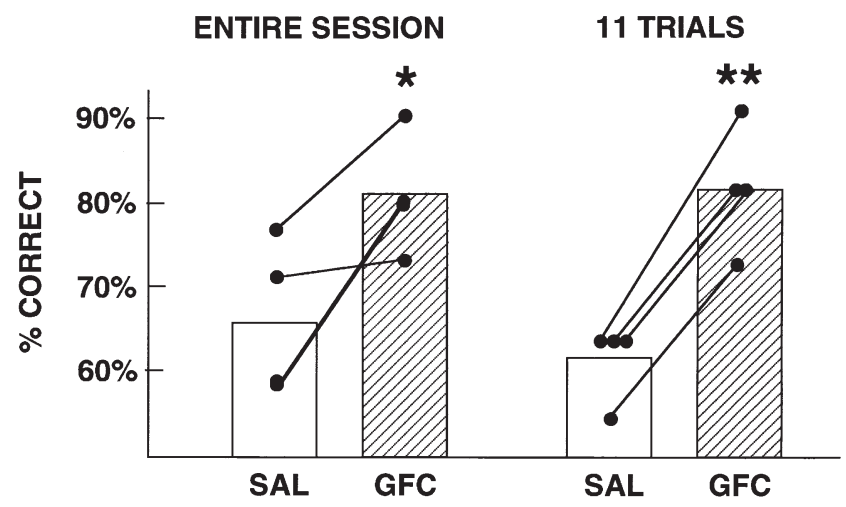

Figure 3. The effects of guanfacine on performance of the delayed response task for the four monkeys. Bar graphs represent mean percent correct following saline (SAL) or guanfacine (GFC) for the entire session (left) or for the 11 trials performed during Tc-99m-ECD infusion (right). The percent correct for each individual monkey is portrayed by a solid circle; the results following saline and guanfacine for each individual monkey are interconnected by a line. All monkeys were improved by guanfacine administration. * indicates different than saline administration $(p=.05) ;{ }^{* *}$ indicates significantly different than saline administration $(p<.003)$. nificance at the 0.05 level (mid vs. caudal dlPFC: $\mathrm{F}(1,3)=$ 8.384, $p=.063$; caudal PFC vs. STG: $\mathrm{F}(1,3)=7.311, p=$ .074 ; mid dlPFC vs. STG: $\mathrm{F}(1,3)=9.956, p=.051)$. However, there was a trend for increasing $\mathrm{rCBF}$ in increasingly posterior cortical regions, such that mid dlPFC < caudal dlPFC $<$ STG (mean relative counts of $137.5<$ $153.3<171.6$ (Figure 4 ).

\section{Effect of Guanfacine}

Planned comparisons showed that guanfacine significantly increased rCBF counts in both PFC regions (mid dlPFC: saline vs. guanfacine $\mathrm{F}(1,3)=12.873, p=.037$; caudal dlPFC: saline vs. guanfacine $\mathrm{F}(1,3)=13.902, p=$ .034), but had no significant effect on rCBF counts in the auditory association cortex (STG: saline vs. guanfacine $\mathrm{F}(1,3)=0.277, p=.635)$. These results are depicted graphically in Figure 4 (relative counts for saline and guanfacine scans) and Figure 5 (percent change between guanfacine and saline scans). Guanfacine increased rCBF in both dIPFC regions in all four monkeys, producing a mean \pm S.E.M. increase in $\mathrm{rCBF}$ of $5.8 \pm 1.4 \%$ for the mid dIPFC and $8.0 \pm 1.7 \%$ for the caudal dlPFC. In contrast, guanfacine had very mixed effects on rCBF in the STG, producing a mean change of only $1.1 \pm 2.9 \%$. The difference between the guanfacine and saline SPECT scans from monkey 1 at the level of the caudal PFC is shown in Figure 2.

\section{Correlation between Drug Effects on Cognitive Performance and SPECT Imaging}

There were high correlations between guanfacine effects on cognitive performance (entire session) and rCBF measures in caudal dlPFC $(r=0.88)$ and mid $\operatorname{dIPFC}(r=0.85)$. However, given the small number of animals in this study, this $r$ value did not achieve significance for a two-tailed test at the 0.05 level $(r=0.95$ required for $\mathrm{df}=2$ ). The correlation between guanfacine's effects on cognitive performance and $\mathrm{rCBF}$ values in STG was somewhat lower $(r=0.71)$. Cognitive data from the 11 trials associated with ECD infusion were not used for correlational analysis given the low level of variability between animals (i.e., three of the four monkeys exhibited an $18.2 \%$ improvement with guanfacine).

\section{DISCUSSION}

The current data replicate previous reports that guanfacine improves spatial working memory performance in young adult monkeys (Franowicz and Arnsten 1998). The current study extended these behavioral findings to ECD SPECT imaging measures of rCBF. Guanfacine administration increased $\mathrm{rCBF}$ values in the mid and caudal dIPFC, the cortical region most associated with 

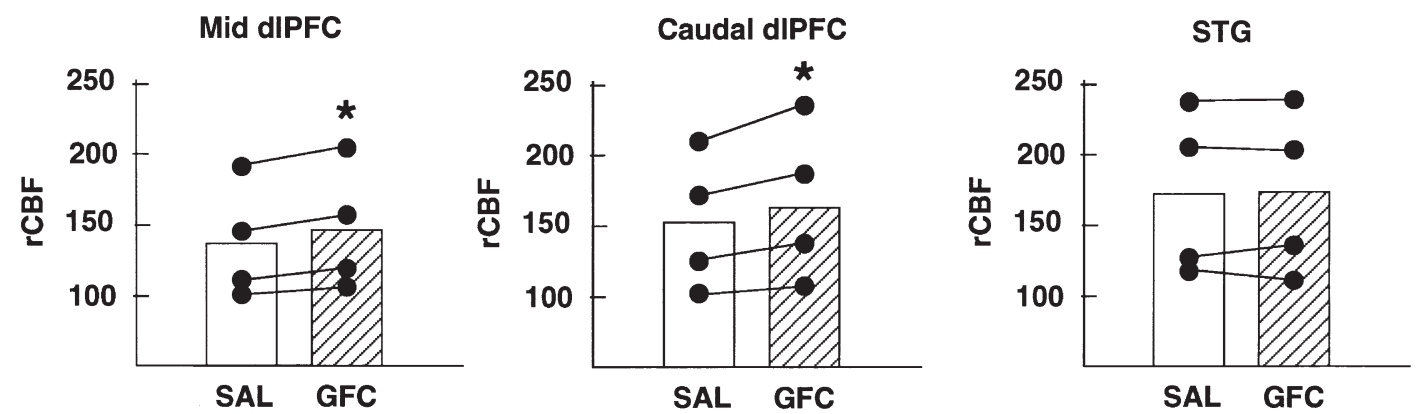

Figure 4. The effects of saline (SAL) vs. guanfacine (GFC) on relative rCBF values in the mid dlPFC, caudal dlPFC, and STG. Bar graphs represent mean counts for the four monkeys. Relative rCBF counts for individual monkeys are represented by solid circles; the saline and guanfacine values for each animal are interconnected by lines. * indicates significantly different than saline $(p<.05)$.

performance of spatial working memory tasks in monkeys (Goldman and Rosvold 1970). rCBF values were increased an average of $8 \%$ in the caudal dlPFC. As epileptic seizures can alter SPECT rCBF values by about $20 \%$ (Spanaki et al. 1999), it can be appreciated that an $8 \%$ increase in $\mathrm{rCBF}$ associated with drug treatment is actually quite substantial. (However, it should be noted that the uptake of ECD is not linearly related to blood flow, but rolls off at higher flow rates.)

Guanfacine had no significant effect on rCBF values in the auditory association cortex (STG), a cortical region which was not relevant to task performance. Thus, the increased $\mathrm{rCBF}$ values in the dIPFC do not appear to be the result of nonspecific increases in rCBF values. Similarly, it is unlikely that guanfacine's hypotensive actions (Arnsten et al. 1988) could induce the pattern of changes in rCBF observed in the current study. The pattern of response is more consistent with regionally selective actions of drug on neural activity. However, it is not known from the current study if guanfacine in-

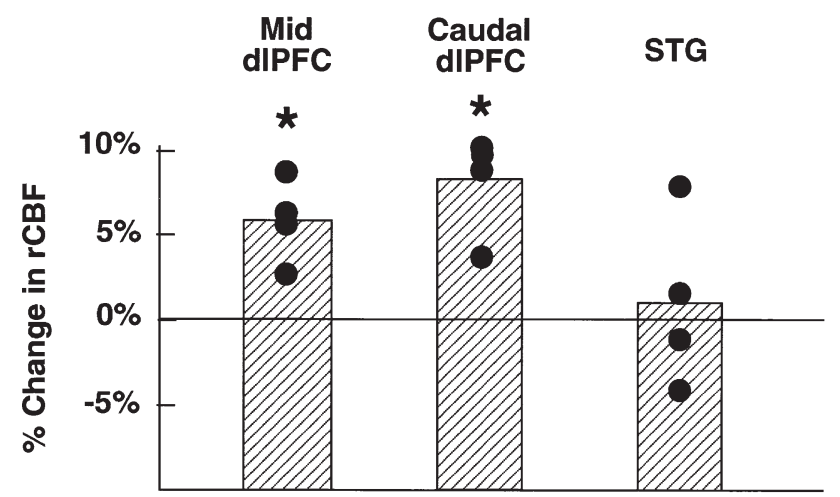

Figure 5. The percent change in rCBF values between saline and guanfacine treatment in the mid dlPFC, caudal dlPFC, and STG. Bar graphs represent mean percent change for the four monkeys; percent changes for individual monkeys are represented by solid circles. * indicates significant difference between saline and guanfacine values $(p<.05)$. creased $\mathrm{rCBF}$ in the dlPFC because the animals were doing a task known to rely on this area, or if guanfacine would increase $\mathrm{rCBF}$ in the dlPFC irrespective of cognitive state. Future monkey imaging studies that examine non-PFC tasks could help clarify guanfacine's contribution to $\mathrm{rCBF}$ changes.

The present study has several limitations which constrain interpretation of the data. Firstly, the small number of subjects used in the study limited statistical power and thus the number of brain regions appropriate for analysis. A larger number of subjects would have allowed us to examine $\mathrm{rCBF}$ changes in brain regions such as the posterior parietal cortex (area 7) and hippocampus relevant to a spatial working memory task. The small number of subjects also limited the power of the correlative analysis between behavioral and SPECT findings. It should be noted that most monkey imaging studies have been limited to small numbers in the range of two to four animals, (e.g., Friedman and Goldman-Rakic 1994). The small number of subjects arises in large part from the extensive time required for adaptation to the challenging procedures necessary for imaging studies, and the difficulty in identifying animals who will perform under these stringent conditions. Studies of this kind are much more feasible in humans for these reasons.

A second limitation in this study is the relatively low resolution of SPECT imaging, particularly when imaging a smaller monkey brain. However, the coregistration of the SPECT scans to the MRI along with use of subtraction image analysis allows homologous regions to be accurately compared and verified against the subject's own anatomy. Although SPECT imaging does not permit the exquisite spatial resolution possible with 2-deoxyglucose (2-DG) autoradiography, resolution is sufficient to document changes in $\mathrm{rCBF}$ in the dorsolateral region of the PFC. The temporal resolution of SPECT is also inferior to functional MRI (fMRI) for measuring rCBF changes, but is sufficient for the generalized changes tested in this study. Future studies using fMRI in humans may 
be able to examine whether guanfacine increases $\mathrm{rCBF}$ in PFC over a long time frame (i.e., producing a state change) or whether increases are specific to times when working memory is engaged (e.g., during the several seconds of the delay period).

The SPECT [or PET] imaging method has many advantages for research in nonhuman primates. The monkey can be tested in the WGTA as usual, and can be infused with the tracer from a distance without the subject being aware of the injection, thus reducing potential disruption. Although fMRI has better temporal resolution, SPECT has the advantage of not requiring the monkey to be awake and immobile in the scanner. SPECT is also noninvasive, and unlike 2-DG studies, monkeys can be used repeatedly as their own controls. This is particularly important for drug studies. SPECT also has some advantages for pharmacological studies in monkeys, where sites of drug action are often determined by infusing drug into a single brain region to see if it effects behavior in a similar fashion to systemic administration. SPECT imaging permits visualization of drug effects on multiple brain regions at once instead of the single region analysis used with infusion studies. For example, in the current study both the PFC and STG were examined, rather than solely focusing on PFC as infusion studies have required. However, the small number of subjects in the current study limited this opportunity.

Finally, SPECT imaging permits examination of drug effects on regions such as the STG which are distant from the cortical surface and therefore very difficult to infuse. It is understood that imaging studies of this kind cannot determine whether a drug is acting directly or indirectly in a brain region to alter rCBF. However, combined with other findings, these data can provide an important corroboration of drug effects on regional brain activity.

One advantage of performing imaging studies in rhesus monkeys is the rich literature on PFC function available for comparison. Extensive lesion and electrophysiological studies have established the dlPFC surrounding the principal sulcus as the critical region for spatial working memory (reviewed in Goldman-Rakic 1987). For example, neurons in this region have been identified which increase their firing rate during the delay period when the spatial position must be remembered (Funahashi et al. 1989). Imaging studies using 2-DG autoradiography have also shown that the principal sulcal region is activated by working memory in rhesus monkeys performing the same delayed response task as used in the current study (Friedman and GoldmanRakic 1994). The results of this 2-DG study showed that working memory processing significantly increased local cerebral glucose utilization by $19 \%$ in the principal sulcus region of the PFC compared to levels during a sensory-motor or discrimination control task (Friedman and Goldman-Rakic 1994). Indeed, more detailed 2-DG studies by Davachi and Goldman-Rakic have highlighted the importance of the medial ventral bank of the principal sulcal cortex as a critical area for both spatial and object working memory (Davachi 1999). This is the same general region demonstrating increased rCBF following guanfacine administration in the current study, although it is understood that the poorer resolution of SPECT imaging does not allow direct comparisons.

A recent infusion study in rhesus monkeys indicates that guanfacine acts directly in this dIPFC region to enhance working memory performance (Mao et al. 1999). Infusions of guanfacine into the caudal two-thirds of the principal sulcal dIPFC produced a delay-related improvement in spatial working memory performance, whereas guanfacine infusions outside this cortical area were ineffective (ibid). Thus, the combined imaging and infusion data reinforce the importance of alpha- 2 adrenoceptor mechanisms to dIPFC working memory function.

\section{Relevance to Neuropsychiatry}

Deficits in PFC cognitive function such as impaired working memory, sustained attention and behavioral inhibition are prominent symptoms in many neuropsychiatric disorders. Alpha-2 agonists such as guanfacine and clonidine are currently being tested in patients with PFC impairment. The alpha-2 agonist, clonidine, has been shown to lessen tics in children with Tourette syndrome (Cohen et al. 1979) and to ameliorate symptoms of ADHD (Hunt et al. 1985). Clonidine has been shown to improve memory and performance of the Stroop interference task in patients with Korsakoff's amnesia (Mair and McEntee 1986) and to enhance memory and Trails B performance in schizophrenic patients (Fields et al. 1988). Most recently, clonidine has been shown to improve word fluency and working memory performance in patients with Alzheimer's Disease (Riekkinen and Riekkinen 1999). Guanfacine has been the focus of more recent research given its superior side effect profile. This drug has been shown to improve symptoms of ADHD and enhance responding on a continuous performance task in open label studies of children with ADHD (Chappell et al. 1995; Horrigan and Barnhill 1995; Hunt et al. 1995). Recent controlled trials in ADHD adults (F. Taylor, personal communication) and children (L. Scahill, personal communication) are also indicating improvement with guanfacine. Thus, alpha-2 agonist treatment may be helpful for treating symptoms of PFC dysfunction in a variety of neuropsychiatric disorders.

\section{ACKNOWLEDGMENTS}

Robert A. Avery was primarily responsible for image analysis; Jenna S. Franowicz was primarily responsible for data collection. 


\section{REFERENCES}

Arnsten AFT (1998): Catecholamine modulation of prefrontal cortical cognitive function. Trends Cogn Sci 2:436-447

Arnsten AFT, Cai JX, Goldman-Rakic PS (1988): The alpha-2 adrenergic agonist guanfacine improves memory in aged monkeys without sedative or hypotensive side effects. J Neurosci 8:4287-4298

Arnsten AFT, Goldman-Rakic PS (1985): Alpha-2 adrenergic mechanisms in prefrontal cortex associated with cognitive decline in aged nonhuman primates. Science 230:1273-1276

Arnsten AFT, Steere JC, Hunt RD (1996): The contribution of a-2 noradrenergic mechanisms to prefrontal cortical cognitive function: Potential significance to Attention Deficit Hyperactivity Disorder. Arch Gen Psychiatry 53:448-455

Cai JX, Ma Y, Xu L, Hu X (1993): Reserpine impairs spatial working memory performance in monkeys: Reversal by the alpha-2 adrenergic agonist clonidine. Brain Res 614:191-196

Carlson S, Ranila H, Rama P, Mecke E, Pertovaara A (1992): Effects of medetomidine, an alpha-2 adrenoceptor agonist, and atipamezole, an alpha-2 antagonist, on spatial memory performance in adult and aged rats. Behav Neural Biol 58:113-119

Chappell PB, Riddle MA, Scahill L, Lynch KA, Schultz R, Arnsten A, Leckman JF, Cohen DJ (1995): Guanfacine treatment of comorbid attention deficit hyperactivity disorder and Tourette's Syndrome: Preliminary clinical experience. J Am Acad Child Adolesc Psychiatry 34:1140-1146

Cohen DJ, Young JG, Nathanson JA, Shaywitz BA (1979): Clonidine in Tourette's Syndrome. Lancet 2:551-553

Coull JT, Frith CD, Dolan RJ, Frackowiak RS, Grasby PM (1997): The neural correlates of the noradrenergic modulation of human attention, arousal and learning. Eur J Neurosci 9:589-598

Coull JT, Middleton HC, Robbins TW, Sahakian BJ (1995): Contrasting effects of clonidine and diazepam on tests of working memory and planning. Psychopharmacology 120: 311-321

Davachi L (1999): Functional Imaging of the Neuronal Networks Underlying Recognition and Working Memory Processes in the Primate Brain: A 2-Deoxyglucose Investigation. Yale University

Fields RB, Van Kammen DP, Peters JL, Rosen J, Van Kammen WB, Nugent A, Stipetic M, Linnoila M (1988): Clonidine improves memory function in schizophrenia independently from change in psychosis. Schizophr Res 1:417-423

Fitzpatrick JM, Hill DLG, Shyr Y, West J, Studholme C, Maurer CR (1998): Visual assessment of the accuracy of retrospective registration of MR and CT images of the brain. IEEE Tran Med Imag 17:571-585

Franowicz JCS, Arnsten AFT (1998): The alpha-2A noradrenergic agonist, guanfacine, improves delayed response performance in young adult rhesus monkeys. Psychopharmacology 136:8-14

Franowicz JS, Arnsten AFT (1999): Treatment with the noradrenergic alpha-2 agonist clonidine, but not diazepam, improves spatial working memory in normal young rhesus monkeys. Neuropsychopharmacology 21:611621

Franowicz JS, Kessler L, Morgan C, Arnsten AFT (1998): The alpha-2 noradrenergic agonist, guanfacine, improves spatial working memory in wild type mice but not mice with point mutations of the gene for the alpha-2A receptor. Soc Neurosci Abstr 24:711

Friedman HR, Goldman-Rakic PS (1994): Coactivation of prefrontal cortex and inferior parietal cortex in working memory tasks revealed by 2DG functional mapping in the rhesus monkey. J Neurosci 14:2775-2788

Funahashi S, Bruce CJ, Goldman-Rakic PS (1989): Mnemonic coding of visual space in the monkey's dorsolateral prefrontal cortex. J Neurophysiol 61:331-349

Goldman PS, Rosvold HE (1970): Localization of function within the dorsolateral prefrontal cortex of the rhesus monkey. Exp Neurol 27:291-304

Goldman-Rakic PS (1987): Circuitry of the primate prefrontal cortex and the regulation of behavior by representational memory. In Plum F (ed), Handbook of Physiology, The Nervous System, Higher Functions of the Brain, Vol. V. Bethesda, MD, American Physiological Society, pp 373-417

Horrigan JP, Barnhill LJ (1995): Guanfacine for treatment of Attention-Deficit-Hyperactivity Disorder in boys. J Child Adolesc Psychopharmacol 5:215-223

Hunt RD, Arnsten AFT, Asbell MD (1995): An open trial of guanfacine in the treatment of attention deficit hyperactivity disorder. J Am Acad Child Adolesc Psychiatry 34:50-54

Hunt RD, Mindera RB, Cohen DJ (1985): Clonidine benefits children with Attention Deficit Disorder and Hyperactivity: Reports of a double-blind placebo-crossover therapeutic trial. J Am Acad Child Psychiatry 24:617-629

Jakala P, Riekkinen M, Sirvio J, Koivisto E, Kejonen K, Vanhanen M, Riekkinen PJ (1999): Guanfacine, but not clonidine, improves planning and working memory performance in humans. Neuropsychopharmacology 20:460-470

Lewis DA, Morrison JH (1989): Noradrenergic innervation of monkey prefrontal cortex: A dopamine-beta-hydroxylase immunohistochemical study. J Comp Neurol 282:317-330

Li B-M, Mao Z-M, Wang M, Mei Z-T (1999): Alpha-2 adrenergic modulation of prefrontal cortical neuronal activity related to spatial working memory in monkeys. Neuropsychopharmacology 21:601-610

Li B-M, Mei Z-T (1994): Delayed response deficit induced by local injection of the alpha-2 adrenergic antagonist yohimbine into the dorsolateral prefrontal cortex in young adult monkeys. Behav Neural Biol 62:134-139

Mair RG, McEntee WJ (1986): Cognitive enhancement in Korsakoff's psychosis by clonidine: A comparison with 1-dopa and ephedrine. Psychopharmacology 88:374-380

Mao Z-M, Arnsten AFT, Li B-M (1999): Local infusion of alpha-1 adrenergic agonist into the prefrontal cortex impairs spatial working memory performance in monkeys. Biol Psychiatry 46:1259-1265

McEntee WJ, Mair RG (1990): The Korsakoff syndrome: A neurochemical perspective. Trends Neurosci 13:340-344 
Moffoot A, O'Carroll RE, Murray C, Dougall N, Ebmeier K, Goodwin GM (1994): Clonidine infusion increases uptake of Tc-exametazime in anterior cingulate cortex in Korsakoff's psychosis. Psychol Med 24:53-61

Riekkinen P, Riekkinen M (1999): THA improves word priming and clonidine enhances fluency and working memory in Alzheimer's Disease. Neuropsychopharmacology 20:357-364

Sawaguchi T (1998): Attenuation of delay-period activity of monkey prefrontal cortical neurons by an alpha-2 adrenergic antagonist during an oculomotor delayedresponse task. J Neurophysiol 80:2200-2205

Spanaki MV, Spencer SS, Corsi M, MacMullan J, Seibyl J, Zubal IG (1999): The sensitivity and specificity of quantitative difference SPECT analysis in seizure localization. J Nucl Med 40:730-736

Studholme C, Hill DLG, Hawkes DJ (1997): Automated three-dimensional registration of magnetic resonance and positron emission tomography brain images by multi-resolution optimisation of voxel similarity measures. Med Physics 24:25-35

Studholme C, Hill DLG, Hawkes DJ (1999): An overlap invariant entropy measure of 3D medical image alignment. Pattern Recogn 32:71-86

Tanila H, Mustonen K, Sallinen J, Scheinin M, Riekkinen P (1999): Role of alpha-2C-adrenoceptor subtype in spatial working memory as revealed by mice with targeted disruption of the alpha-2C-adrenoceptor gene. Eur J Neurosci 11:599-603

Tanila H, Rama P, Carlson S (1996): The effects of prefrontal intracortical microinjections of an alpha-2 agonist, alpha-2 antagonist and lidocaine on the delayed alternation performance of aged rats. Brain Res Bull 40:117119

Uhlen S, Wikberg JES (1991): Delineation of rat kidney alpha 2A and alpha 2B-adrenoceptors with [3H]RX821002 radioligand binding: Computer modeling revels that guanfacine is an alpha-2A-selective compound. Eur J Pharmacol 202:235-243

Walovitch RC, Hill TC, Garrity ST, Cheesman EH, Burgess BA, O'Leary DH, Watson AD, Ganey MV, Morgan RA, Williams SJ (1989): Characterization of Technetium99m-L,L-ECD for brain perfusion imaging. Part 1: Pharmacology of Technetium-99m ECD in nonhuman primates. J Nucl Med 30:1892-1901

West J, Fitzpatrick JM, Wang MY, Dawant BM, Maurer CR, Kessler RM, Maciunas RJ, Barillot C, Lemoine D, Collignon A, Maes F, Suetens P, Vandermeulen $D$, van den Elsen PA, Napel S, Sumanaweera TS, Harkness B, Hemler PF, Hill DLG, Hawkes DJ, Studholme C, Maintz JBA, Viergever MA, Malandain G, Pennec X, Noz ME, Maquire GQ, Pollack M, Pelizzari CA, Robb RA, Hanson D, Woods RP (1997): Comparison and evaluation of retrospective intermodality image registration techniques. J Comput Assist Tomogr 21:554-566 